\title{
Respostas Endócrinas e Ovarianas Associadas com o Folículo Dominante da Primeira Onda Folicular em Ovelhas Sincronizadas com CIDR ou PGF ${ }_{2 \alpha}{ }^{1}$
}

\section{Luis Fernando Uribe-Velásquez ${ }^{2}$, Eunice Oba ${ }^{3}$, Lida Constanza Lara-Herrera ${ }^{4}$, Maria Inês Lenz Souza ${ }^{5}$, Hooverman Villa-Velásquez ${ }^{4}$, Luzia Aparecida Trinca ${ }^{6}$, Carlos Antônio de Carvalho Fernandes ${ }^{7}$}

\begin{abstract}
RESUMO - Os efeitos de prostaglandina $\left(\mathrm{PGF}_{2}\right.$ a) vs CIDR e eCG (gonadotrofina coriônica eqüina) na dinâmica folicular da primeira onda e sua relação com as concentrações plasmáticas de $\mathrm{P}_{4}$ e $\mathrm{E}_{2}$ foram investigadas em ovelhas cíclicas. Foram utilizadas 14 fêmeas ovinas da raça Bergamascia; o Grupo 1 (G1) foi submetido a duas aplicações de PGF $2 \alpha$ e o Grupo 2 (G2), tratado com CIDR durante 14 dias, sendo que, no momento de sua retirada, administraram-se 500 UI de eCG. A dinâmica folicular ovariana foi monitorada por meio de ultrasom. Monitoraram-se todos os folículos $\geq 2 \mathrm{~mm}$ e mapeou-se sua posição diariamente, observando-se o desenvolvimento individual de cada folículo. Desde o dia anterior à aplicação da segunda dose de $\mathrm{PGF}_{2 \alpha}(\mathrm{G} 1)$ e desde a administração de eCG (G2) até o décimo dia do ciclo estral, foram coletadas amostras de sangue para análise de $\mathrm{P}_{4}$ e $\mathrm{E}_{2}$. Houve diferença significativa nas concentrações plasmáticas de $\mathrm{P}_{4}$ e $\mathrm{E}_{2}$ entre os tratamentos. A sincronização de estro e ovulação, utilizando CIDR + 500 UI de eCG, incrementou a quantidade de folículos recrutados, além de aumentar o diâmetro máximo e a taxa de crescimento dos folículos grandes na primeira onda folicular.
\end{abstract}

Palavras-chave: dinâmica folicular, estradiol $\left(\mathrm{E}_{2}\right)$, ovinos, progesterona $\left(\mathrm{P}_{4}\right)$, ultra-sonografia

\section{Endocrine and Ovarian Response Associated with the First-Wave Follicle Dominant in Sheep Synchronized either CIDR or $\mathbf{P G F}_{2 \alpha}$}

\begin{abstract}
The effect of prostaglandin $\left(\mathrm{PGF}_{2} \mathrm{a}\right)$ vs CIDR and eCG (equine chorionic gonadotrophin) on follicular dynamic of wave 1 and its relation with $\mathrm{P}_{4}$ and $\mathrm{E}_{2}$ plasma concentrations were investigated in cyclic ewes. Fourteen Bergamascia ewes were used, Group 1 (G1) synchronized by two i.m. injections of $\mathrm{PGF}_{2 \alpha}$ and Group 2 (G2) treated for $14 \mathrm{~d}$ with CIDR and 500 IU of eCG at dispositive withdrawal on day 14. Ovarian follicular dynamics were ultrasonically monitored. All the ovarian follicles $\geq 2 \mathrm{~mm}$ were measured, and their relative locations were recorded on an ovarian map in order to follow the sequential development of each individual follicle. Blood samples for $\mathrm{P}_{4}$ and $\mathrm{E}_{2}$ determination were collected daily from $1 \mathrm{~d}$ before the second injection of $\mathrm{PGF} 2 \alpha(\mathrm{G} 1)$ and administration of eCG (G2) until day 10 after ovulation of the cycle. There were significant differences in $\mathrm{P}_{4}$ and $\mathrm{E}_{2}$ plasma concentrations between treatments. The CIDR + eCG (500 IU) treatment enhanced the recruitment of smaller follicles and enhanced the maximum diameter and growth rate of large follicles during wave 1.
\end{abstract}

Key Words: follicular dynamics, oestradiol $\left(\mathrm{E}_{2}\right)$, ovine, progesterone $\left(\mathrm{P}_{4}\right)$, ultrasonography

\section{Introdução}

Em um programa de manejo reprodutivo ovino, as vantagens do uso de inseminação artificial dependem do controle de estro e da ovulação. Os métodos mais utilizados para a indução e sincronização de estro e estimulação do crescimento folicular em ovelhas envolvem progesterona (P4) e/ou progestágenos e a administração intramuscular de eCG (gonadotrofina sérica de égua gestante). Entretanto, a primeira barreira é a diminuição na taxa de fertilidade, que está estreitamente relacionada com a grande variabilidade no tempo e no número de ovulações, sendo que parte dessa variação pode ser atribuída à quantidade total de folículos em crescimento presentes no ovário antes do tratamento (Noel et al., 1994).

A sincronização de estro, segundo Gonzáles de Bulnes et al. (1994), desencadeia diversas mudanças

\footnotetext{
${ }_{1}^{1}$ Projeto financiado pela FAPESP, São Paulo, Brasil.

${ }^{2}$ Professor do Departamento de Saúde Animal, Faculdade de Ciências Agropecuárias, Universidad de Caldas, Manizales, Caldas, Colômbia. E.mail: luisfuribe@andinet.com

${ }^{3}$ Professor da Faculdade de Medicina Veterinária e Zootecnia, FMVZ, UNESP, Botucatu, SP.

${ }_{5}^{4}$ Médico - Residente de Faculdade de Medicina, UNESP, Botucatu, SP.

${ }^{5}$ Bolsista de Pós-Doutorado da FAPESP, FMVZ, UNESP, Botucatu, SP. E.mail: mlenz@zipmail.com.br

${ }^{6}$ Professor do Instituto de Biociências, UNESP, Botucatu, SP.

${ }^{7}$ Professor da Faculdade de Medicina Veterinária, UNIFENAS, Alfenas, MG.
} 
na dinâmica folicular em ruminantes. Uma variável a ser medida seria a relação existente entre os folículos e o estágio folicular no início do tratamento em ovelhas, assim como a presença de poucos folículos pequenos ou a permanência estável de um folículo $>5 \mathrm{~mm}$ por um período mínimo de dois dias, no início do protocolo.

Outra alternativa para sincronização de estro são as prostaglandinas $\left(\mathrm{PGF}_{2 \alpha}\right)$, visando a formulação de sistemas simples e de curta duração. $\mathrm{A} \mathrm{PGF}_{2 \alpha}$ é o fator luteolítico que induz a regressão prematura do corpo lúteo por meio da interrupção da fase progestacional do ciclo estral, iniciando, assim, novo ciclo (Herrera et al., 1990), fenômeno demonstrado pela persistência do corpo lúteo frente à imunização ativa e passiva contra $\mathrm{PGF}_{2 \alpha}$.

Naqvi et al. (1998) reportaram uma eficiente sincronização de estro administrando doses de $10 \mathrm{ou}$ 7,5 mg de $\mathrm{PGF}_{2 \alpha}$ em um intervalo de 10 dias, independentemente do dia do ciclo estral em ovelhas Kheri. Pesquisando a resposta das ovelhas à administração de $\mathrm{PGF}_{2 \alpha}$ do segundo ao 15 o dia do ciclo estral, Hackett \& Robertson (1980) verificaram menor resposta nos dias 2 - 3 do ciclo estral (20\%) mas, quando as doses eram aumentadas nestes dias, a resposta atingiu $100 \%$. Portanto, os investigadores concluíram que, no início do ciclo estral, o tecido luteal em formação é mais resistente à luteólise provocada pela $\mathrm{PGF}_{2 \alpha} \mathrm{e}$, devido à meia-vida curta da $\mathrm{PGF}_{2 \alpha}$, pequenas doses repetidas deste hormônio podem ter o mesmo efeito de uma única dose elevada. Por outro lado, a associação de $\mathrm{PGF}_{2 \alpha}$ e gonadotrofinas tipo eCG aumentou significativamente a taxa ovulatória e o número de ovulações duplas em ovelhas (McNatty et al., 1982).

Quando da administração de $\mathrm{P}_{4}$ exógena ou seus análogos (como o acetato de melengestrol-MAP) por 12 ou 14 dias, ocorreu a regressão do corpo lúteo, mas o estro e a ovulação apenas manifestaram-se quando a $\mathrm{P}_{4}$ exógena foi removida (Castonguay et al., 1990). Os mesmos autores, utilizando esponjas impregnadas com MAP por 14 dias para sincronização de estro em ovelhas mestiças Boorola com Finnish (BFL), mestiças Boorola com Suffolk (BS), Finnish Landrace (FINN) e Suffolk (S) determinaram, por intermédio de laparotomia no dia da retirada do pessário, mudanças na população folicular. A quantidade média de folículos $>4 \mathrm{~mm}$ foi maior $(3,5 \pm 0,3)$ nas ovelhas Finish Landrace do que nas outras raças. Não houve diferenças na quantidade média dos folículos de 3 - $4 \mathrm{~mm}$ entre os diferentes grupos genéticos $(2,9 \pm 0,6 ; 2,4 \pm 0,3$;
$2,6 \pm 0,4 ;$ e $1,8 \pm 0,5$, respectivamente). As ovelhas mestiças Boorola x Suffolk $(35,8 \pm 3,1)$; Suffolk $(35,1 \pm 2,7)$; e mestiças Boorola x Finish $(32,9 \pm 3,1)$ apresentaram mais folículos pequenos $(1-3 \mathrm{~mm})$ do que as ovelhas Finish Landrace $(24,9 \pm 2,2)$. Na raça Suffolk os folículos maiores mediram 7,6 $\pm 0,3 \mathrm{e}$ $5,8 \pm 0,4 \mathrm{~mm}$ para $\mathrm{F} 1$ (folículo dominante) e $\mathrm{F}_{2}$ (folículo subordinado), respectivamente.

Nesse sentido, Godfrey et al. (1995), avaliando o uso do CIDR e da $\mathrm{PGF}_{2 \alpha}$ em ovelhas, concluíram que as fêmeas tratadas com o CIDR apresentaram o estro bem mais cedo que os animais tratados com $\mathrm{PGF}_{2 \alpha}$ (1,4 $\pm 0,4$ e 2,9 \pm 0,4 dias, respectivamente), enquanto as concentrações séricas de $\mathrm{P}_{4}$ no décimo dia após o estro foram semelhantes para os dois grupos.

A eCG deve estar associada ao CIDR para estimular a ovulação, não só na estação reprodutiva, como fora dela, uma vez que, na ausência de eCG, não há manifestação de estros (Rubianes et al., 1998). Assim, Evans \& Robinson (1980) constataram que o uso isolado de eCG, em altas doses, produz uma resposta menos eficiente que quando o hormônio é combinado com os progestágenos exógenos, observando neste caso melhor resposta na fertilidade. Além disso, os dados obtidos por Cardwell et al. (1998), em ovelhas mestiças Dorset com Rambouillet, indicaram que o início de estro e a ovulação se manisfestaram mais rapida e uniformemente, como resultado da combinação do progestágeno com a eCG.

A eCG interfere no eixo hipotalâmico-hipofisárioovariano e nos mecanismos regulatórios intraovarianos, devido à sua vida média longa e a sua atividade de FSH e LH. Assim, estas propriedades não são somente benéficas, mas também têm seus efeitos negativos na seqüência normal dos eventos fisiológicos do desenvolvimento folicular e maturação oocitária (Donrov et al., 1998).

Dria ncourt et al. (1993) relataram que as gonadotrofinas de tipo eCG podem afetar os mecanismos responsáveis pelo crescimento folicular e aumentar a taxa ovulatória, pela redução no diâmetro de folículos menores $(0,8 \mathrm{~mm}$ na ovelha e $1,5 \mathrm{~mm}$ em bovinos), ou pelo recrutamento de folículos de menor diâmetro.

A terapia de progestágenos, segundo Mihm et al. (1996), requer a suplementação de um estimulante hormonal do tipo gonadotrofina coriônica eqüina (eCG), para aumentar a produção de estrogênio pelo folículo. O uso de eCG em conjunto com progestágenos, conforme constatado por Greyling e Brink (1987), aumenta o índice de resposta das 
fêmeas ovinas à sincronização de estro, reduzindo o intervalo entre a remoção do implante e a ovulação. Turnbull et al. (1977) constataram um aumento na quantidade de folículos $>0,5 \mathrm{~mm}$ após aplicação de eCG em ovelhas Merino, sendo ainda maior o aumento no número dos folículos > $3,5 \mathrm{~mm}$, com poucos folículos em estágio atrésico. O mesmo tipo de observações foi descrito por Dott et al. (1979), porém os folículos atrésicos $(2-2,9 \mathrm{~mm})$ eram menores no diâmetro.

Aplicando eCG no dia zero do ciclo estral em ovelhas (dia da ovulação), Noel et al. (1994) constataram, por laparoscopia, que houve um aumento no recrutamento dos folículos pequenos e na taxa de ovulação. Comparando também a sincronização usando apenas eCG vs progestágenos por 14 dias mais a eCG em ovelhas, durante a estação reprodutiva, Evans \& Robinson (1980) não encontraram efeitos significativos no desenvolvimento folicular total, corpo lúteo e as concentrações plasmáticas de $\mathrm{P}_{4}$ e $\mathrm{E}_{2}$, observando também que a administração excessiva da eCG (800 a 1000 UI) resultou em luteinização prematura, devido aos efeitos luteinizantes da eCG, somados à grande quantidade de $\mathrm{E}_{2}$ produzido pelos folículos grandes (> $5 \mathrm{~mm}$ ), estimulando prematuramente a hipófise, com a conseqüente liberação de LH. Além disso, segundo os autores o aumento das concentrações plasmáticas de $\mathrm{P}_{4}$ seria resultado do aumento da estimulação do corpo lúteo e do folículo.

Segundo Leyva et al. (1998), a concentração plasmática de progesterona $\left(\mathrm{P}_{4}\right)$ elevou-se no dia seguinte da ovulação $(0,8 \mathrm{ng} / \mathrm{mL})$, mas só significativa a partir do segundo dia $(2,4 \pm 0,3 \mathrm{ng} / \mathrm{mL})$. As concentrações de $\mathrm{P}_{4}$ e o diâmetro do corpo lúteo aumentaram até $\mathrm{o}$ sexto ou sétimo dia do ciclo estral $(7,6 \pm 0,4 \mathrm{ng} / \mathrm{mL}$ e 11,4 $\pm 0,2 \mathrm{~mm}$, respectivamente); a partir daí, as duas variáveis foram inconstantes até o dia 10. Depois do dia 11, as duas medidas começaram a decrescer, mas o declínio de $\mathrm{P}_{4}$ foi mais acentuado, quando comparado com o diâmetro do corpo lúteo.

Acritopoulou et al. (1977), comparando um grupo de ovelhas sincronizadas com uma injeção de $\mathrm{PGF}_{2 \alpha}$ (100 mg) e outro grupo em condições naturais, não observaram diferenças na concentração plasmática de $\mathrm{P}_{4}$ durante os períodos de pré e pós-tratamento. Depois da injeção de cloprostenol, um análogo sintético de $\mathrm{PGF}_{2 \alpha}$, em ovelhas Finn-Merino, Campbell et al. (1990) notaram que as secreções de $E_{2}$ e de androstenediona elevaram-se nas quatro ou oito horas seguintes. Após este aumento, no início da fase folicular, a secreção de $\mathrm{E}_{2}$ continuou elevando-se até o aumento na concentração de LH; paralelamente, a concentração plasmática de $\mathrm{P}_{4}$ diminuiu até valores basais.

O presente trabalho foi conduzido com o objetivo de avaliar os efeitos da sincronização de estro com $\mathrm{PGF}_{2 \alpha}$ vs o CIDR + 500 UI de eCG no desenvolvimento da primeira onda folicular em fêmeas ovinas, na população folicular e nas concentrações plasmáticas de $\mathrm{P}_{4}$ e $\mathrm{E}_{2}$.

\section{Material e Métodos}

O trabalho experimental foi desenvolvido na FMVZ/UNESP-Botucatu. Utilizaram-se 14 fêmeas ovinas adultas da raça Bergamácia, durante a estação reprodutiva, com peso corporal de $60,42 \pm 8,16 \mathrm{~kg}$, com idades de dois a cinco anos, previamente examinadas quanto ao estado clínico geral, sanitário e reprodutivo. Os animais foram mantidos em boxes de $3 \times 3 \mathrm{~m}$, sob luminosidade natural. As fêmeas foram distribuídas em dois grupos, o Grupo um $(\mathrm{n}=7)$ submetido a duas aplicações de PGF $_{2 \alpha}$ (125 mg; CiosinR), com intervalo de nove dias, e o Grupo $2(n=7)$ tratado com o dispositivo intra-vaginal por 14 dias (CIDR contendo 0,3g de progesterona, AHI Plastic Moulding Company, Hamilton, NZ), sendo que no momento de sua remoção administraram-se 500 UI de eCG via intramuscular (PMSG-CAL 5000 UI). As fêmeas foram mantidas com um macho vasectomizado para observacão da manifestação de estro. A alimentação consistiu de uma ração concentrada ( $20 \%$ de proteína bruta e $5,05 \%$ de fibra bruta) e feno de Cynodon dactylon (L.) "Coastcross" (4,98\% de proteína bruta e 35,88\% de fibra bruta, na matéria seca). Os animais receberam 1,5 kg/cabeça/dia de concentrado e 2,0 kg/ cabeça/dia de feno, com disponibilidade ad libitum de mistura mineral e água.

As ovelhas, em posição de estação, foram monitoradas por meio de ultra-som (SSD-500; Aloka Co. Ltda, Japão) com um transdutor linear prostático humano de 7,5 Mhz (Modelo UST-660-7,5; Aloka Co. Ltda, Japão). O dia da ovulação (dia zero) definiu-se a partir do momento do desaparecimento do maior folículo $\ell 5 \mathrm{~mm}$ ). As imagens ecográficas foram armazenadas em vídeo e desenharam-se diagramas da posição de todos os folículos $\geq 2 \mathrm{~mm}$.

Desde o dia anterior à aplicação da segunda dose de $\mathrm{PGF}_{2 \alpha}$ (Grupo 1) e administração de eCG (Grupo 2) até o décimo dia do ciclo estral, coletaram-se amos tras 
de sangue cada dia, às $8 \mathrm{~h}$, por venopunção jugular armazenando-se e o plasma obtido a $-20^{\circ} \mathrm{C}$, para determinação de $\mathrm{P}_{4}$ e $\mathrm{E}_{2}$. A dosagem foi feita por radioimunoensaio (RIA) no Laboratório de Endocrinologia do Departamento de Reprodução Animal e Radiologia Veterinária da UNESP-Botucatu, utilizando-se kits comerciais Coat-A-Count (Diagnostic Products Corporation, Los Angeles, CA, USA) fase sólida, usando-se um contador gama Vitek, modelo Kineti Count 48. Os resultados obtidos através da contagem do contador gama foram transformados em unidades de medida característica do respectivo hormônio, com base nas amostras padrões, por intermédio de um programa computacional em Gwbasic. A sensibilidade do ensaio para $\mathrm{P}_{4}$ e $\mathrm{E}_{2}$ foi de $0,1 \mathrm{ng} / \mathrm{mL}$ e $8 \mathrm{pg} / \mathrm{mL}$, respectivamente. Para as concentrações plasmáticas de $\mathrm{P} 4$ utilizaram-se três controles contendo baixa, média e alta concentração de $\mathrm{P}_{4}$ : $20,68 \%(0,29 \pm 0,06 \mathrm{ng} / \mathrm{mL}) ; 12,28 \%(2,28 \pm 0,28 \mathrm{ng} / \mathrm{mL})$; e $2,70 \%(5,93 \pm 0,16 \mathrm{ng} / \mathrm{mL})$. Todas as amostras de $\mathrm{P}_{4}$ foram analisadas num só ensaio. $\mathrm{O}$ coeficiente intraensaio para as concentrações plasmáticasde $E_{2}$ foi de $15,87 \%(21,99 \pm 3,49 \mathrm{pg} / \mathrm{mL})$ e o coeficiente de variação inter-ensaio de 4,53\% $(22,72 \pm 1,03 \mathrm{pg} / \mathrm{mL})$. Os efeitos dos tratamentos nas concentrações plasmáticas dos hormônios foram determinados pela análise de medidas repetidas utilizando-se o programa Statistical Analysis System (Latour \& Littlel, 1996). Para os efeitos fixos (tratamento, dia, tratamento x dia) foi utilizado o teste $\mathrm{F}$, e para as comparações múltiplas das médias usou-se o teste de tukey-kramer. Para a análise da taxa de crescimento e a população folicular total, usou-se ANOVA e as médias foram comparadas pelo teste F. Os folículos classificaram-se como pequenos $(2-2,5 \mathrm{~mm})$, médios $(3-3,5 \mathrm{~mm})$ e grandes (= $4 \mathrm{~mm})$. As médias da população dos folículos pequenos, médios e grandes foram transformadas antes de serem submetidas à ANOVA, e as médias, comparadas pelo teste $\mathrm{F}$.

\section{Resultados e Discussão}

Observou-se, por intermédio da técnica ultrasonográfica, pelo menos um corpo lúteo na metade da fase luteal em todos os animais. Independentemente do programa de sincronização utilizando prostaglandina $\left(\mathrm{PGF}_{2 \alpha}\right)$ ou CIDR, seguido da administração de 500 UI de eCG (gonadotrofina coriônica eqüina) intramuscular, na retirada do dispositivo, as concentrações plasmáticas de $\mathrm{P}_{4}$ foram menores que
$1 \mathrm{ng} / \mathrm{mL}$ nas 24 horas seguintes ao tratamento, indicando que a luteólise foi rápida e completa.

Os padrões de crescimento do folículo dominante durante a primeira onda, foram diferentes nos animais controle, quando comparados àqueles tratados com CIDR e 500 UI de eCG (Tabela 1).

Durante a sincronização de estro no grupo experimental, o fato de as fêmeas encontrarem-se em diferentes fases do ciclo estral, quando o dispositivo contendo $\mathrm{P}_{4}$ (CIDR) foi inserido, induziu a variação entre os animais na duração da secreção de $\mathrm{P}_{4}$ endógena. Assim, conforme as observações de Leyva et al. (1998), essas mudanças resultantes da combinação de $\mathrm{P}_{4}$ exógena e endógena podem provocar alterações na dinâmica folicular, e variações na apresentação de estro após a retirada do dispositivo.

$\mathrm{O}$ dia de emergência da primeira onda folicular não foi diferente entre os dois grupos experimentais, sendo de $0,57 \pm 0,53$ e $0,86 \pm 0,40$, para os grupos controle e tratado com a gonadotrofina exógena, respectivamente, valores próximos aos obtidos por Noel et al. (1993) e Leyva et al. (1998).

O folículo dominante atingiu seu diâmetro máximo de 4,29 $\pm 0,26 \mathrm{~mm}$ no dia 4,57 $\pm 0,43$ nos animais controle e, $5,36 \pm 0,26 \mathrm{~mm}$ no dia $5,71 \pm 0,52$ nos animais submetidos à administração de CIDR + eCG, apresentando-se diferença estatisticamente signifi-

Tabela 1 -Folículo dominante da primeira onda folicular em ovelhas Bergamácia sincronizadas com CIDR + eCG (500 UI) durante a estação reprodutiva (médias $\pm E P$ )

Table 1 - Dominant follicle of wave 1 in Bergamácia ewes synchronized with CIDR + eCG (500 UI) during breeding season (means $\pm E P$ )

\begin{tabular}{lcc}
\hline $\begin{array}{l}\text { Variáveis } \\
\text { Variables }\end{array}$ & $\begin{array}{c}\text { Controle } \\
\text { Control }\end{array}$ & $\begin{array}{c}\text { Tratadas- } \mathrm{P}_{4} \\
\text { (CIDR) } \\
\text { Treated-P4 } \\
(\text { CIDR) }\end{array}$ \\
\hline $\begin{array}{l}\text { Dia de emergência } \\
\text { Emergence day }\end{array}$ & $0,57 \pm 0,53^{\mathrm{a}}$ & $0,86 \pm 0,40^{\mathrm{a}}$ \\
$\begin{array}{l}\text { Dia do máximo diâmetro } \\
\text { Maximum diameter (day) }\end{array}$ & $4,57 \pm 0,43^{\mathrm{c}}$ & $5,71 \pm 0,52^{\mathrm{d}}$ \\
$\begin{array}{l}\text { Diâmetro máximo (mm) } \\
\text { Maximum diameter (mm) }\end{array}$ & $4,29 \pm 0,26^{\mathrm{c}}$ & $5,36 \pm 0,26^{\mathrm{d}}$ \\
$\begin{array}{l}\text { Dias do platô } \\
\text { Static phase (days) } \\
\text { Taxa de crescimento (mm/dia) } \\
\text { Growing rate (mm/day) }\end{array}$ & $1,00 \pm 0,09^{\mathrm{a}}$ & $1,12 \pm 0,07^{\mathrm{b}}$ \\
\hline
\end{tabular}

Médias seguidas da mesma letra na linha não diferem estatisticamente $a$ vs $b \quad(\mathrm{p}<0,001) ; c$ vs $d \quad(\mathrm{p}<0,05)$.

Means followed by the same letter do not differ statistically a vs $b(p<.001)$; $c$ vs $d \quad(p<.05)$. 
cativa para as duas variáveis citadas $(p<0,05)$. O diâmetro máximo atingido pelo folículo dominante nos animais controle esteve de acordo com os valores observados por Ravindra et al. (1994) e Rubianes et al. (1996), sendo menor que os encontrados por Ginther et al. (1995) e Leyva et al. (1998).

A administração da eCG no momento de retirada do dispositivo, possivelmente diminuiu os efeitos negativos de $\mathrm{P} 4$ na dinâmica de crescimento folicular e ovulação. O crescimento do folículo dominante nos animais tratados, foi significativamente maior que no grupo controle, concordando com os valores mostrados por Leyva et al. (1998).

O aumento do diâmetro folicular do folículo dominante nos animais recebendo eCG pode ter sido provocado pelos efeitos da gonadotrofina no eixo hipotálamo-hipófise-ovário e pelas alterações nos mecanismos regulatórios intraovarianos, devido, principalmente, à sua meia-vida prolongada e pela sua atividade semelhante ao LH e ao FSH, assim como constatado por McNeilly et al. (1991).

Os dias de platô não apresentaram diferença significativa para os grupos experimentais, sendo para os animais controle e tratados, respectivamente de $1,29 \pm 0,18$ e 1,57 $\pm 0,20$, similares aos resultados demonstrados por Rubianes et al. (1996).

A taxa de crescimento folicular foi estatisticamente diferente $(\mathrm{p}<0,001)$ para os grupos controle e tratados, com valores de 1,0 $\pm 0,09$ e 1,12 $\pm 0,07$ dias, respectivamente. Os valores observados para a taxa de crescimento estão de acordo com os resultados obtidos no trabalho de Castonguay et al. (1990) em ovelhas (0,9 a 1,7 mm/dia). Leyva et al. (1998) sugeriram que a taxa de crescimento em alguns folículos, quando comparado com outros que crescem mais rapidamente, pode desencadear fortes implicações na qualidade do oócito no momento da ovulação.

De acordo com as observações do experimento, fica evidente que o crescimento folicular é um processo contínuo e independente da fase do ciclo estral (McNeilly et al., 1991), e o padrão de crescimento folicular em ondas faz com que a população de folículos de diversas classes de tamanhos altere-se ao longo do ciclo (Pierson \& Ginther, 1987).

A distribuição (médias $\pm \mathrm{EP}$ ) dos folículos pequenos, médios e grandes nas fêmeas controle e sincronizadas com CIDR + eCG (500 UI) é apresentada, respectivamente, nas Figuras 1, 2 e 3.

Durante o início e metade da fase luteal, observou-se diariamente crescimento dos folículos peque- nos (Figura 1), concordando com as observações de Schrick et al. (1993) em ovelhas Suffolk. Ravindra et al. (1994) também verificaram o aparecimento de folículos $>2 \mathrm{~mm}$ em todos os dias, exceto para os dias $1,5,15,16$ e 17 do intervalo interovulatório em fêmeas ovinas mestiças White-Faced. No presente experimento, constatou-se que o recrutamento dos folículos destinados a ovular é realizado a partir dos folículos > $2 \mathrm{~mm}$ no momento da luteólise.

Houve interação significativa entre tratamento $\mathrm{x}$ dias do ciclo estral ( $p<0,05)$. A aplicação de eCG (500 UI) provocou aumento significativo dos folículos pequenos nos dias -2, -1 pré-ovulatórios e no dia 6 após ovulação, apresentando $9,0 \pm 1,21$ e 3,43 $\pm 0,30$ $(\mathrm{p}<0,001) ; 8,43 \pm 0,97$ e 4,29 $\pm 1,06(\mathrm{p}<0,05) ;$ e 8,43 $\pm 1,36$ e $4,43 \pm 0,30$ folículos $(\mathrm{p}<0,05)$, respectivamente, para os animais tratados e controle.

$\mathrm{O}$ aumento do recrutamento folicular, assim como a manutenção destes novos folículos no grupo tratado, pode ter resultado das mudanças mediadas pelo progestágeno na freqüência dos pulsos de LH. Entretanto, a quantidade de folículos pequenos diminuiu nos animais controle nos dias que antecederam a ovulação, achado semelhante ao constatado por Ravindra et al. (1994), provavelmente devido ao efeito inhibitório do folículo dominante no crescimento dos outros folículos presentes no ovário.

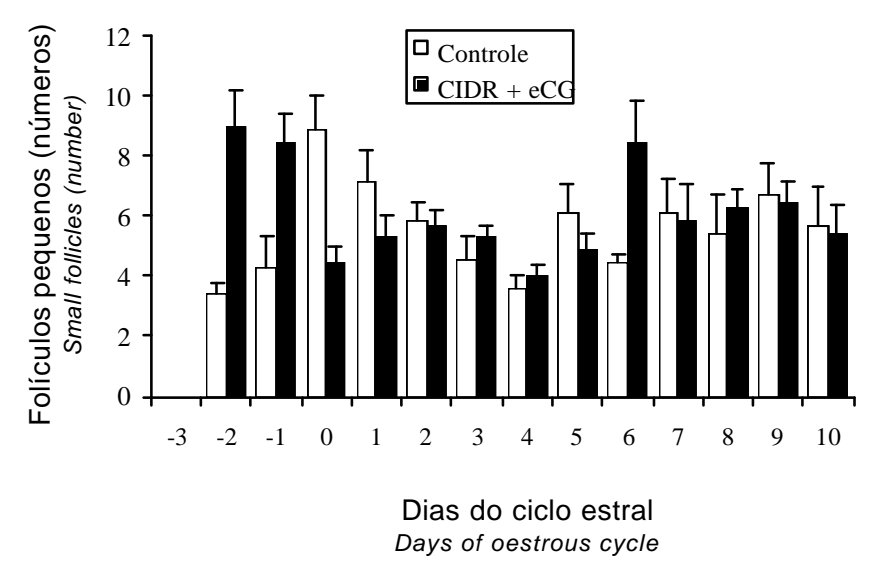

Figura 1 - Distribuição dos folículos pequenos (médias \pm EP dos dados transformados) durante a fase luteal em ovelhas Bergamácia sincronizadas com CIDR + eCG (500 UI) durante a estação reprodutiva.

Figure 1 - Distribution of small follicles (means \pm EP of transformed dates) during luteal phase in Bergamácia ewes synchronized with CIDR + eCG (500 UI) during breeding season. 


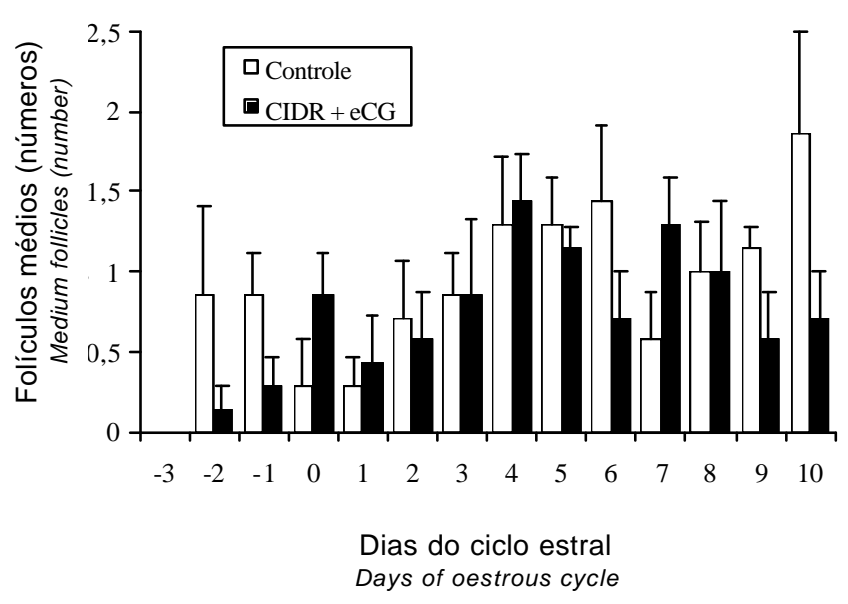

Figura 2 - Distribuição dos folículos médios (médias \pm EP dos dados transformados) durante a fase luteal em fêmeas ovinas Bergamácia sincronizadas com CIDR + eCG (500 UI) durante a estação reprodutiva.

Figure 2 - Distribution of medium follicles (means \pm EP of transformed dates) during luteal phase in Bergamacia ewes synchronized with CIDR + eCG (500 UI) during the breeding season.

A quantidade média dos folículos pequenos (Figura 1) assemelhou-se aos achados de Noel et al. (1994), utilizando laparoscopia, os quais observaram que, em ovelhas Suffolk, a aplicação de 800 UI de eCG depois da retirada do pessário intravaginal (40 mg de FGA) aumentou a população dos folículos pequenos durante a fase folicular, os quais diminuíram no início e metade da fase luteal. Observações do aumento no recrutamento de folículos $>2 \mathrm{~mm}$ após aplicação de eCG, foram também descritas por Dott et al. (1979).

Houve interação significativa entre tratamento e dias do ciclo estral $(\mathrm{p}<0,05)$ para os folículos médios. Os efeitos caracterizaram-se pelo aumento significativo dos folículos médios na metade da fase luteal (Figura 2). O tratamento com progestágeno seguido de eCG provocou aumento significativo $(\mathrm{p}<0,01)$ no número de folículos médios, os quais cresceram nos dias zero $(0,86 \pm 0,26$ e $0,29 \pm 0,29$ folículos $)$ e sétimo $(1,29$ $\pm 0,29$ e $0,57 \pm 0,30$ folículos), respectivamente, para as fêmeas tratadas e controle, seguidos por uma gradativa diminuição do número dos folículos médios no grupo tratado. Possivelmente, a maior quantidade de folículos médios após ovulação pode ser atribuída ao maior pool de folículos presentes nos dias -2 e -1 pré-ovulatórios, como também pode ter sido indicador da prevenção do processo normal de atresia folicular, exercida pela administração de eCG.

R. Bras. Zootec., v.31, n.2, p.944-953, 2002 (suplemento)

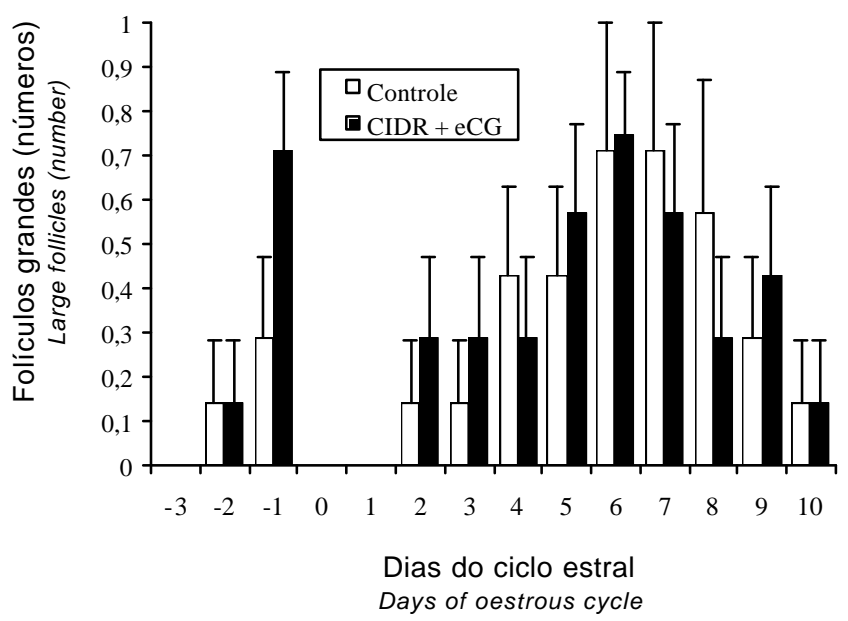

Figura 3 - Distribuição dos folículos grandes (médias \pm EP dos dados transformados) no início e metade da fase luteal em fêmeas ovinas Bergamácia sincronizadas com CIDR + eCG (500 UI) durante a estação reprodutiva.

Figure 3 - Distribution of large follicles (means $\pm E P$ of transformed dates) during luteal phase in Bergamacia ewes synchronized with CIDR + eCG (500 UI) during the breeding season.

Nesse sentido, foi exposto por Mariana \& Machado (1976) que a eCG provoca a formação do antro folicular em folículos pequenos em fêmeas bovinas, incrementando a taxa de secreção e a multiplicação das células da granulosa, causando o aumento do diâmetro da população folicular. Assim, Driancourt et al. (1991) destacaram que o número de receptores para LH em folículos menores tratados com a gonadotrofina é maior, quando comparados com folículos grandes em fêmeas bovinas cíclicas. Além disso, esses autores sugeriram que o aumento no crescimento folicular pode ser resultante do incremento da acumulação do fluido antral.

A quantidade média dos folículos grandes (Figura 3) não apresentou diferença estatística significativa entre os dois grupos de animais estudados, ainda que a quantidade dos folículos $\geq 4 \mathrm{~mm}$ tenha sido superior nas fêmeas tratadas com o CIDR seguido da gonadotrofina, no dia -1 antes da ovulação $(0,29 \pm$ $0,18$ e $0,71 \pm 0,18$ folículos $)$ e no segundo $(0,14 \pm 0,14$ e $0,29 \pm 0,18)$, terceiro $(0,14 \pm 0,14$ e $0,29 \pm 0,18)$, quinto $(0,43 \pm 0,20$ e $0,57 \pm 0,20)$ e nono dias $(0,29 \pm$ 0,18 e $0,43 \pm 0,20$ folículos) após ovulação, respectivamente, para os grupos controle e tratados.

Resultados semelhantes foram encontrados por Noel et al. (1994) e Leyva et al. (1998), que verificaram um número significativamente maior de folículos 
grandes em ovelhas Suffolk, administrando respectivamente, CIDR + 750 UI de eCG ou $40 \mathrm{mg}$ de FGA +800 UI de eCG.

Entretanto, uma pesquisa desenvolvida por Castonguay et al. (1990) destacou que, possivelmente, um número significativo de folículos apresentando diâmetro de $1-3 \mathrm{~mm}$, pode induzir a atresia dos folículos grandes em fêmeas ovinas.

A sincronização utilizada não influenciou significativamente na quantidade média da população folicular total no início e metade da fase luteal do ciclo estral (Figura 4), entretanto houve diferença significativa $(p<0,05)$ entre os dias experimentais, sendo que também foi constatada diferença significativa na interação tratamento $x$ dia $(\mathrm{p}<0,05)$.

Assim, no presente protocolo, o início do tratamento no começo do desenvolvimento da primeira onda folicular, provocou maior recrutamento dos folículos $>2 \mathrm{~mm}$, observado principalmente nos dias $-2(4,43 \pm 0,53$ e $9,29 \pm 1,21$ folículos totais $)$ e $-1(5,43 \pm 1,17$ e 9,43 $\pm 0,97$ folículos totais $)$ antes da ovulação, nos grupos controle e tratados, respectivamente, achados que concordam as observações feitas por Rubianes et al. (1997) em fêmeas Corriedale. Esses resultados mostram que, em ovelhas Bergamácia, o crescimento de folículos antrais $>2$

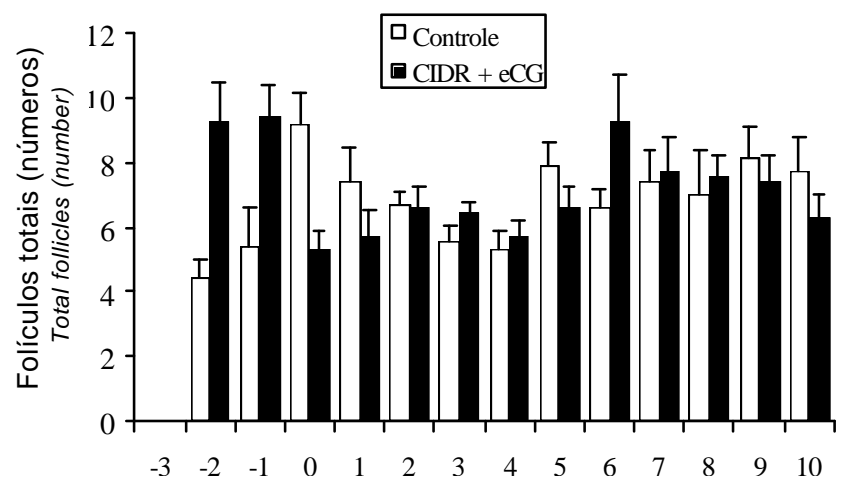

Dias do ciclo estral Days of oestrous cycle

Figura 4 - Distribuição da população folicular (médias \pm EP) durante a fase luteal em ovelhas Bergamácia sincronizadas com CIDR + eCG (500 UI) durante a estação reprodutiva.

Figure 4 - Distribution of follicular population (means \pm EP of transformed dates) during luteal phase in Bergamácia ewes synchronized with CIDR + eCG (500 UI) during the breeding season.

R. Bras. Zootec., v.31, n.2, p.944-953, 2002 (suplemento) $\mathrm{mm}$, acontece em todos os dias, durante o início e metade da fase luteal do ciclo estral. Durante cada onda de crescimento, folículos $>2 \mathrm{~mm}$ desenvolveram-se de umpoolrecrutado e só alguns continuaram seu crescimento e maturação. Também tem sido constatado, em fêmeas bovinas, que o início do tratamento superovulatório na presença de um folículo grande melhora a resposta folicular ao tratamento (Fortune, 1993).

A sincronização do estro incrementou a quantidade de folículos recrutados, além de aumentar o diâmetro máximo e a taxa de crescimento dos folículos grandes na primeira onda folicular quando comparado com a taxa de crescimento durante o ciclo normal, corroborando as hipóteses expostas por Driancourt et al. (1991) e Noel et al. (1994), pesquisando bovinos e ovinos, respectivamente. Entretanto, tem sido apontado por outros investigadores (Turnbull et al., 1997) que o aumento na taxa ovulatória originada da administração de eCG não é correlacionado com o número médio da população antral, mas sim com a quantidade de folículos viáveis de 0,8 a 2 mm de diâmetro.

A concentração de progesterona $\left(\mathrm{P}_{4}\right)$ plasmática (médias $\pm \mathrm{EP}$ ) do grupo controle e as fêmeas sincronizadas estão apresentados na Figura 5. A concentração plasmática de $\mathrm{P} 4$ não foi diferente entre os dois grupos experimentais, nos dias $-2 \mathrm{e}-1$ antes da ovulação $(0,36 \pm 0,06$ e $0,48 \pm 0,03 \mathrm{ng} / \mathrm{mL} ;$ e $0,22 \pm 0,06$ e $0,26 \pm 0,03 \mathrm{ng} / \mathrm{mL}$, respectivamente). Por outro lado, as fêmeas sincronizadas apresentaram um leve aumento nas concentrações de $\mathrm{P}_{4}$ após a retirada do dispositivo e aplicação de eCG, fato que pode atrasar o início da liberação das gonadotrofinas.

Os valores de concentrações plasmáticas de $\mathrm{P} 4$ antes da ovulação, na presente pesquisa, estão de acordo com os resultados reportados por Scaramuzzi et al. (1993), que observaram valores abaixo de $1 \mathrm{ng} / \mathrm{mL}$ durante a fase folicular.

Os animais do tratamento controle apresentaram concentrações circulantes de $\mathrm{P}_{4}$ baixas no primeiro dia após ovulação $(0,20 \pm 0,33 \mathrm{ng} / \mathrm{mL})$, com um aumento significativo no quarto dia $(2,05 \pm 0,33 \mathrm{ng} / \mathrm{mL})$. As concentrações continuaram aumentando progressivamente do quinto $(3,43 \pm 0,33 \mathrm{ng} / \mathrm{mL})$ ao nono dia $(4,91 \pm 0,33 \mathrm{ng} / \mathrm{mL})$, evidenciando diferença estatística significativa $(\mathrm{p}<0,0001)$ entre os dias do ciclo estudados. Valores inferiores foram reportados por Schrick et al. (1993), devido principalmente ao tipo de ensaio utilizado. 
Houve diferença significativa $(\mathrm{p}<0,01)$ nas concentrações plasmáticas de $\mathrm{P}_{4}$ entre os tratamentos, na metade da fase luteal. Do mesmo modo, também foi constatada diferença significativa entre dias $(\mathrm{p}<0,0001)$ e na interação tratamento e dias do ciclo estral $(\mathrm{p}<0,05)$.

Os animais sincronizados apresentaram aumento significativo $(\mathrm{p}<0,01)$ nas concentrações plasmáticas de P4 desde o sexto até o décimo dia após ovulação, quando comparados aos animais controle. Estes resultados corroboram os de Mihm et al. (1996), os quais mostraram que a $\mathrm{P}_{4}$ fornecida pelo dispositivo exerceu um efeito de feedback negativo inibindo a liberação de $\mathrm{LH}$.

$\mathrm{O}$ aumento nas concentrações plasmáticas de $\mathrm{P}_{4}$ nesse período pode ser o resultado da atividade do corpo lúteo somado à atividade de folículos grandes luteinizados. Portanto, o eCG pode provocar hiperestimulação folicular, resultando em maior tamanho do corpo lúteo, evidenciando sua atividade luteotrófica (Evans \& Robinson, 1980).

Segundo Pearce \& Robinson (1985), em um experimento com ovelhas Merino, houve aumento linear na taxa ovulatória quando aumentaram a dose de eCG de 300 para 600 UI. De forma experimental semelhante, Oyedipe et al. (1989) observaram elevação também nas concentrações plasmáticas de $\mathrm{P}_{4}$ em ovelhas Yankasa estabelecendo, possivelmente, a existência de uma relação direta entre a dosagem

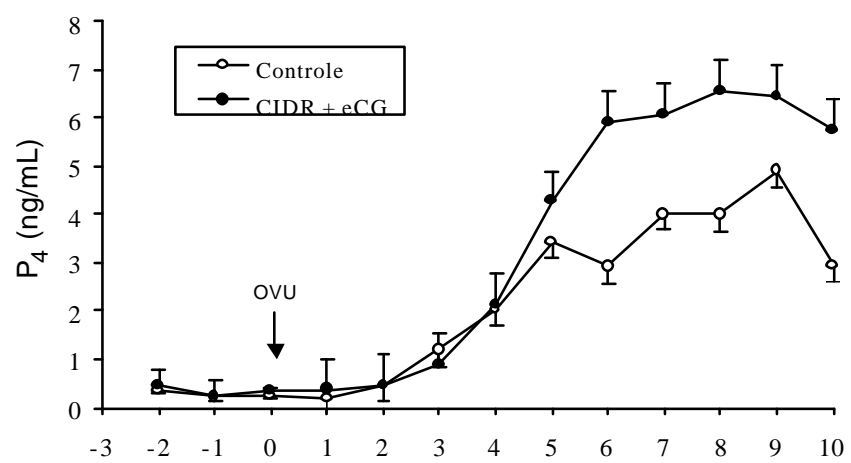

Dias do ciclo estral

Days of oestrous cycle

Figura 5 - Concentrações plasmáticas de progesterona (médias $\pm E P$ ) em ovelhas Bergamácia sincronizadas com CIDR + eCG (500 UI) durante a estação reprodutiva.

Figure 5 - Plasma progesterone concentrations (means $\pm E P$ ) in Bergamácia ewes synchronized with CIDR + eCG (500 UI) during the breeding season.

R. Bras. Zootec., v.31, n.2, p.944-953, 2002 (suplemento) de eCG, corpos lúteos, taxa ovulatória, e concentrações plasmáticas de $\mathrm{P}_{4}$ e $\mathrm{E}_{2}$, corroborando os achados de Evans \& Robinson (1980).

$\mathrm{O}$ perfil das concentrações de estradiol $\left(\mathrm{E}_{2}\right)$ plasmático (médias $\pm \mathrm{EP}$ ) nos grupos experimentais está apresentado na Figura 6 . As concentrações de $\mathrm{E}_{2}$ no sangue foram estatisticamente diferentes $(\mathrm{p}<0,0001)$ entre o grupo controle e o grupo sincronizado com CIDR + eCG, com valores de 8,34 $\pm 0,63$ e 10,61 $\pm 0,63$ pg $/ \mathrm{mL}$ nos dias -2 e -1 , respectivamente, para os animais controle, enquanto os animais tratados apresentaram valores de $15,50 \pm 1,75$ e $17,10 \pm 1,75 \mathrm{pg} / \mathrm{mL}$, respectivamente para os dias -2 e -1 pré-ovulatórios. Os resultados são próximos aos encontrados por Campbell et al. (1990) e Ravindra et al. (1994). As médias foram próximas às reportadas por Pearce e Robinson (1985), os quais constataram que a aplicação de eCG incrementa a resposta ovariana, evidenciada pela quantidade de folículos desenvolvidos.

As concentrações de $\mathrm{E}_{2}$ elevaram-se depois da regressão luteal em associação com o crescimento do folículo dominante. Também foram obtidos por Scaramuzzi et al. (1993) e Schrick et al. (1993) aumentos nas concentrações de $\mathrm{E}_{2}$ plasmático nos dias três a oito; entretanto, foram descritos aumentos significativos por Baird et al. (1976) nos dias seis a nove do ciclo estral, o que pode estar ligado, estreitamente, ao desenvolvimento das ondas de crescimento folicular.

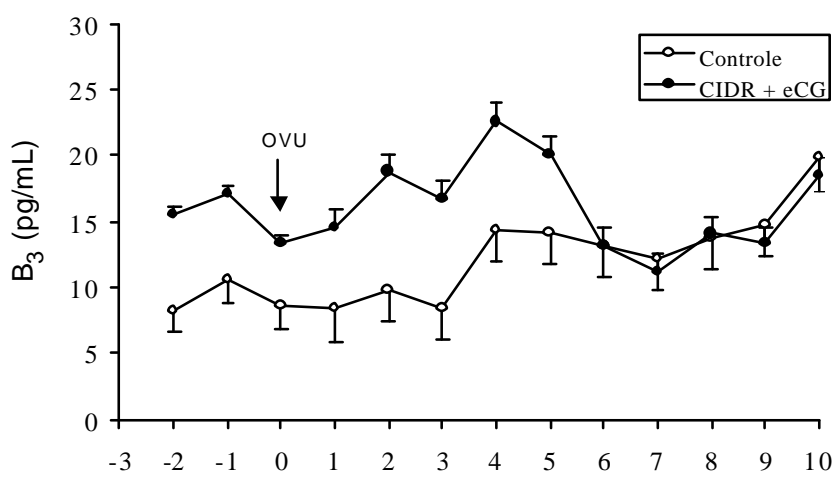

Dias do ciclo estral Days of oestrous cycle

Figura 6 - Concentrações plasmáticas de estradiol (médias $\pm E P)$ em ovelhas Bergamácia sincronizadas com CIDR + eCG (500 UI) durante a estação reprodutiva.

Figure 6 - Plasma estradiol concentrations (means $\pm E P$ ) in Bergamácia ewes synchronized with CIDR + eCG (500 UI) during the breeding season. 
Os resultados corroboram os achados de Kouskoura et al. (1995), os quais mostraram que a sincronização de estro acompanhada da administração de eCG (8 UI/kg), na retirada do implante de $\mathrm{P}_{4}$ (375 mg), em ovelhas Friesland, Chios, Serres e Karagouniki, provocou aumentos nas concentrações plasmáticas de $\mathrm{E}_{2}$ nas quatro horas seguintes e, a partir daí, diminuíram gradualmente, o que revelou significativas diferenças estatísticas entre raças, sugerindo também que o uso de menor dosagem de eCG, no momento da retirada do progestágeno, permite melhor e mais precisa sincronização de estro e ovulação. Também foram reportados aumentos nas concentrações plasmáticas de $\mathrm{E}_{2}$ após retirada do dispositivo contendo a $\mathrm{P}_{4}$, no pró-estro e estro, em ovelhas Rambouillet pesquisadas pelo mesmo autor.

Nos animais controle, o $\mathrm{E}_{2}$ plasmático manteve-se baixo no início da fase luteal, com valores semelhantes aos verificados por Pant et al. (1977) e Scaramuzzi \& Radford (1983). O padrão de secreção mostrou um valor de $8,74 \pm 1,35 \mathrm{pg} / \mathrm{mL}$ no primeiro dia após ovulação, com apresentação de dois aumentos significativos no quarto $(14,42 \pm 1,35 \mathrm{pg} / \mathrm{mL})$ e no décimo dia $(19,84 \pm 1,35 \mathrm{pg} / \mathrm{mL})$, o que evidenciou uma diferença significativa $(\mathrm{p}<0,001)$ entre os dias do ciclo estral, no início e metade da fase luteal. Estes dados assemelharam-se aos de Baird e Scaramuzzi (1976), refletindo, possivelmente, o aumento na atividade folicular.

Houve diferença significativa $(p<0,001)$ nas concentrações plasmáticas de $\mathrm{E}_{2}$ entre os tratamentos após o dia da ovulação, sendo ainda constatada diferença significativa entre os dias $(p<0,001)$ e na interação tratamento e dia $(\mathrm{p}<0,05)$.

As fêmeas ovinas sincronizadas apresentaram elevadas concentrações plasmáticas de $\mathrm{E}_{2}$ no início da fase luteal (Figura 6), atingindo 14,53 $\pm 2,44 \mathrm{pg} / \mathrm{mL}$ no primeiro dia do ciclo estral, com apresentação de dois aumentos significativos no quarto $(22,58 \pm 2,44 \mathrm{pg} / \mathrm{mL})$ e no décimo dias $(16 \pm 2,44 \mathrm{pg} / \mathrm{mL})$. Dessa forma, os achados para as concentrações plasmáticas de $\mathrm{E}_{2}$ mostraram-se semelhantes aos reportes de Sánchez et al. (1995) em fêmeas bovinas, sincronizadas com $\mathrm{P}_{4}$ exógena, nas quais a elevação nas concentrações plasmáticas de $\mathrm{E}_{2}$ foi associada com o incremento no tamanho do folículo dominante concluindo, então, que leves aumentos na freqüência dos pulsos de LH podem promover o crescimento folicular ovariano e dominância, associados com altas concentrações de $\mathrm{E}_{2}$ no plasma.

R. Bras. Zootec., v.31, n.2, p.944-953, 2002 (suplemento)

\section{Conclusões}

A sincronização de estro e ovulação em fêmeas ovinas, utilizando o CIDR e 500 UI de eCG, incrementa a quantidade de folículos recrutados, além de aumentar o diâmetro máximo e a taxa de crescimento dos folículos grandes na primeira onda de desenvolvimento folicular.

A associação CIDR +500 UI de eCG causa aumentos significativos nas concentrações plasmáticas de progesterona $\left(\mathrm{P}_{4}\right)$ e estradiol $\left(\mathrm{E}_{2}\right)$ no início da fase luteal em fêmeas ovinas.

\section{Literatura Citada}

ACRITOPOUlOU, S.; HARESIGN, W.; FOSTER, J.P. et al. Plasma progesterone and LH concentrations in ewes after injection of an analogue of prostaglandin F-2a Journal of Reproduction and Fertility, v.49, p.337-340, 1977.

BAIRD, D.T.; LAND, R.B.; SCARAMUZZI, R.J. et al. Endocrine changes associated with luteal regression in the ewe: the secretion of ovarian oestradiol, progesterone and androstenedione and uterine prostaglandin F2a throught the oestrus cycle. Journal of Endocrinology, v.69, p.275-286, 1976.

BAIRD, D.T.; SCARAMUZZI, R.J. The source of ovarian oestradiol and androstenedione in the sheep during the luteal phase. Acta Endocrinological, v.83, p.402-409, 1976.

CAMPBELL, B.K.; MANN, G.E.; McNEILLY, A.S. et al. The pattern of ovarian inhibin, estradiol, and androstenedione secretion during the estrous cycle of the ewe.Endocrinology, v.127, p.227-235, 1990.

CARDWELL, B.E.; FITCH, G.Q.; GEISERT, R.D. Ultrasonic evaluation for the time of ovulation in ewes treated with norgestomet and norgestomet followed by pregnant mare's serum gonadotropin. Journal of Animal Science, v.76, p.2235-2238, 1998.

CASTONGUAY, F.; DUFOUR, J.J.; MINVIELlE, F. et al. Follicular dynamics and dominance in Boorola $\mathrm{x}$ Finnish Landrace and Boorola $x$ Suffolk ewes heterozygous for the $F$ gene. Journal of Reproduction and Fertility, v.89, p.193-203, 1990.

DONROV, T.S.; BATSAIHAN, D.; LEY, W.B. Gonadotrophin extraction from pregnant mare's serum and effect of PMSG preparation on the fertility of Mongolian native ewes. Small Ruminant Research, v.28, p.61-66, 1998.

DOTT, H.M.; HAY, M.F.; CRAN, D.G. et al. Effect of exogenous gonadotrophin (PMSG) on the antral follicle population in the sheep. Journal of Reproduction and Fertility, v.56, p.683-689, 1979.

DRIANCOURT, M.A.; THATCHER, W.W.; TERQUI, M. Dynamics of ovarian follicular development in cattle during the estrous cycle, early pregnancy and in response to PMSG. Domestic Animal Endocrinology, v.8, p.209-221, 1991.

DRIANCOURT, M.A.; GOUGEON, A.; ROYÈRE, D. et al. Ovarian function. In: THIBAULT, C.; LEVASSEUR, M.C. (Eds.) Reproduction in mammals and man. Amsterdam: Elsevier, 1993. p.283-305. 
EVANS, G.; ROBINSON, T.J. The control of fertility in sheep: endocrine and ovarian responses to progestagen-PMSG treatment in the breeding season and in anoestrus. Journal of Agricultural Science, v.94, p.69-88, 1980.

FORTUNE, J.E. Follicular dynamics during the bovine estrous cycle: A limiting factor in improvement of fertility?Animal Reproduction Science, v.33, p.111-125, 1993.

GINTHER, O.J.; KOT, K.; WILTBANK, M.C. Associations between emergence of follicular waves and fluctuations in FSH concentrations during the estrous cycle in ewes. Theriogenology, v.43, p.689-703, 1995.

GODFREY, R.W.; GRAY, L.; COLliNS, J.R. Estrus synchronization of sheep in the tropics using either controlled internal drug release (CIDR) dispensers or prostaglandin $\mathrm{F}_{2 \alpha}$ (PGF). Journal of Animal Science, v.73, p.232, 1995.

GONZÁLEZ DE BULNES, A.; MORENO, J.S.; GARCIA, L.M. et al. Observación del ovario en la oveja y eficacia en la detección de folículos y cuerpos lúteos mediante ecografía transrectal. Investigación Agraria - Producion y Sanidad Animal, v.9, p.319-329, 1994.

GREYLING, J.P.C.; BRINK, W.C.J. Synchronization of oestrus in sheep: The use of controlled internal drug release (CIDR) dispensers. South Afrikan Tydskr Veekderman, v.17, p.128-132, 1987.

HACKETT, A.J.; ROBERTSON, H.A. Effect of dose and time of injection of prostaglandin $\mathrm{F}_{2 \alpha}$ in cycling ewes. Theriogenology, v.13, p.347-351, 1980.

HERRERA, H.L.; FELDMAN, S.D.; ZARCO, Q.L. et al. Evaluación del efecto luteolítico de la prostaglandina $\mathrm{F}_{2}$ alfa en diferentes días del ciclo estral de la borrega. Veterinaria México, v.21, p.143-147, 1990.

KOUSKOURA, T.H.; KOUIMTZIS, S.; ALEXAKI, E. et al. 1995. Comparative studies of ovarian steroids in blood, and specific proteolytic enzymes in the cervical mucus, in four sheep breeds after oestrus synchronization (Progesterone and PMSG). 1. Breed variation of oestradiol-17 $\beta$ and progesterone in blood during natural oestrus, synchronization oestrus, and the first oestrus after synchronized oestrus. Reproduction in Domestic Animals, v.30, p.8-13, 1995.

LATOUR, D.; LITTELL, R.Advanced general linear models with an emphasis on mixed models. Version 6.12. Cary: SAS Institute, 1996. 268p.

LEYVA, V.; BUCKRELL, B.C.; WALTON, J.S. Regulation of follicular and ovulation in ewes by exogenous progestagen. Theriogenology, v.50, p.395-416, 1998.

MARIANA, J.C.; MACHADO, J. Etude de la formation de l'antrum dans les follicles de l'ovaire de ratte et de vache normales ou stimulées par PMSG. Annual Biological Animal Biochemistry and Biophysics, v. 16, p.545-559, 1976.

McNATTY, K.P.; GIBB, M.; DOBSON, C. et al. Preovulatory follicular development in sheep treated with PMSG and/or prostaglandin. Journal of Reproduction and Fertility, v.65, p.111-123, 1982.

McNEILlY, A.S.; PICTON, H.M.; CAMPBELL, B.K. et al. Gonadotrofic control of follicle growth in the ewe. Journal of Reproduction and Fertility, v.43, p.177-186, 1991.

MIHM, M.; DISKIN, M.G.; ROCHE, J.F. Regulation of follicle wave growth in cattle.Reproduction in Domestic Animals, v.31, p.531-538, 1996.

NAQVI, S.M.K.; GULYANI, R.; PMITTAL, J. Estrus synchronization response in Kheri ewes treated with prostaglandin $F_{2} \alpha$. Indian Journal of Animal Science, v.68, p.564-565, 1998.

NOEL, B.; BISTER, J.L.; PAQUAY, R. Ovarian follicular dynamics in Suffolk ewes at differents periods of the year. Journal of Reproduction and Fertility, v.99, p.695-700, 1993.

R. Bras. Zootec., v.31, n.2, p.944-953, 2002 (suplemento)
NOEL, B.; BISTER, J.L.; PIERQUIN, B. et al. Effects of FGA and PMSG on follicular growth and LH secretion in Suffolk ewes. Theriogenology, v.41, p.719-727, 1994.

OYEDIPE, E.O.; PATHIRAJA, N.; GYANG, E.O. et al. Effect of dose of pregnant mare serum gonadotrophin on estrus parameters, ovulation rate and peripheral progesterone concentrations in Yankasa ewes. Animal Reproduction Science, v.20, p.255-264, 1989.

PANT, H.C.; HOPKINSON, C.R.N.; FITZPATRICK, R.J. Concentration of oestradiol, progesterone, luteinizing hormone and follicle-stimulating hormone in the jugular venous plasma of ewes during the oestrus cycle. Journal of Endocrinology, v.73, p.247-255, 1977.

PEARCE, D.T.; ROBINSON, T.J. Plasma progesterone concentrations, ovarian and endocrinological responses and sperm transport in ewes with synchronized oestrus.Journal of Reproduction and Fertility, v.75, p.49-62, 1985.

PIERSON, R.A.; GINTHER, J.O. Follicular populations during the estrous cycle in heifers. I - Influence of day. Animal Reproduction Science, v.14, p.165-176, 1987.

RAVINDRA, J.P.; RAWLINGS, N.C.; EVANS, A.C.O. et al. Ultrasonographic study of ovarian follicular dynamics in ewes during the estrous cycle.Journal of Reproduction and Fertility, v.101, p.501-509, 1994.

RUBIANES, E.; CASTRO, T.; CARBAJAL, B. Effect of high progesterone levels during the growing phase of the dominant follicle of wave 1 in ultrasonically monitored ewes. Canadian Journal of Animal Science, v.76, p.1-3, 1996.

RUBIANES, E.; UNGERFELD, R.; VIÑOLES, C. et al. Ovarian response to superstimulatory treatment initiated relative to wave emergency in ultrasonographically monitored ewes. Theriogenology, v.47, p.1479-1488, 1997.

RUBIANES. E.; CASTRO, T.; KMAID, S. Estrus response after a short progesterone priming in seasonally anestrous goats. Theriogenology, v.49, p.356-362, 1998.

SÁNCHEZ, T.; WEHRMAN, M.E.; KOJIMA, F.N. et al. Dosage of the synthetic progestin, norgestomet, influences luteinizing hormone pulse frequency and endogenous secretion of 17-b-estradiol in heifers.Biology of Reproduction, v.52, p.464-469, 1995.

SCARAMUZZI, R.J.; ADAMS, N.R.; BAIRD, D.T., et al. A model for follicle selection and the determination of ovulation rate in the ewe. Reproduction Fertility and Development, v.5, p.459-478, 1993.

SCARAMUZZI, R.J.; RADFORD, H.M. Factors regulating ovulation rate in the ewe. Journal of Reproduction and Fertility, v.69, p.353-367, 1983.

SCHRICK, F.N.; SURFACE, R.A.; PRITCHARD, J.Y. et al. Ovarian structures during the estrous cycle and early pregnancy in ewes. Biology of Reproduction, v.49, p.1133-1140, 1993.

TURNBUlL, K.E.; BRADEN, A.W.H.; MATTNER, P.E. The pattern of follicular growth and atresia in the ovine ovary. Australian Journal of Biological Sciences, v.30, p.229-241, 1977.
Recebido em: 07/08/00

Aceito em: 14/01/02 